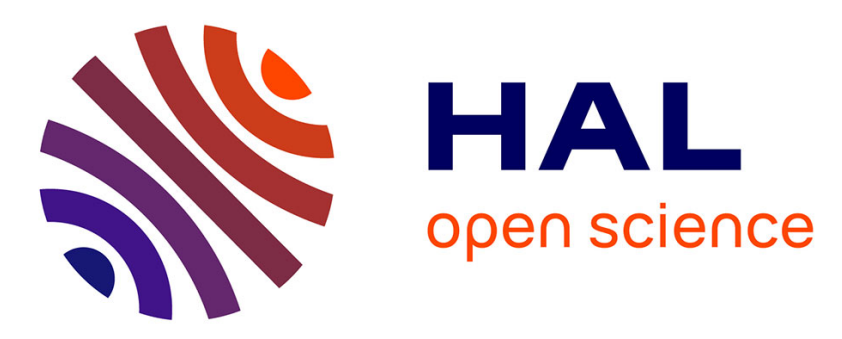

\title{
Estimating motion between avian vertebrae by contact modeling of joint surfaces
}

Matthieu Furet, Anick Abourachid, Christine Böhmer, Valentine Chummun, Christine Chevallereau, Raphaël Cornette, Xavier de La Bernardie, Philippe Wenger

\section{To cite this version:}

Matthieu Furet, Anick Abourachid, Christine Böhmer, Valentine Chummun, Christine Chevallereau, et al.. Estimating motion between avian vertebrae by contact modeling of joint surfaces. Computer Methods in Biomechanics and Biomedical Engineering, 2022, 25 (2), pp.123-131. 10.1080/10255842.2021.1934676 . hal-03344918

\author{
HAL Id: hal-03344918 \\ https://hal.science/hal-03344918
}

Submitted on 15 Sep 2021

HAL is a multi-disciplinary open access archive for the deposit and dissemination of scientific research documents, whether they are published or not. The documents may come from teaching and research institutions in France or abroad, or from public or private research centers.
L'archive ouverte pluridisciplinaire HAL, est destinée au dépôt et à la diffusion de documents scientifiques de niveau recherche, publiés ou non, émanant des établissements d'enseignement et de recherche français ou étrangers, des laboratoires publics ou privés. 


\title{
Estimating motion between avian vertebrae by contact modeling of joint surfaces
}

\author{
Matthieu Furet ${ }^{\mathrm{a}}$, Anick Abourachid ${ }^{\mathrm{b}}$, Christine Böhmer ${ }^{\mathrm{b}}$, Valentine Chummun ${ }^{\mathrm{b}}$, \\ Christine Chevallereau ${ }^{\mathrm{a}}$, Raphaël Cornette ${ }^{\mathrm{b}}$, Xavier De La Bernardie ${ }^{\mathrm{c}}$, Philippe \\ Wenger $^{\mathrm{a}}$ \\ ${ }^{a}$ UMR 6004, CNRS, Laboratoire des Sciences du Numérique de Nantes (LS2N), Ecole \\ centrale de Nantes, 44321 Nantes, France ; ${ }^{\text {b }}$ UMR 7179 CNRS/MNHN, Département \\ Adaptations du Vivant, Muséum National d'Histoire Naturelle, 55 rue Buffon, 75005 Paris, \\ France ; ${ }^{\mathrm{c}}$ UMR 6457 , Subatech, Laboratoire de physique subatomique et des technologies \\ associées, La Chantrerie, 44307 Nantes, France
}

\section{ARTICLE HISTORY \\ Compiled June 20, 2021}

\begin{abstract}
Estimating the motion between two bones is crucial for understanding their biomechanical function. The vertebral column is particularly challenging because the vertebrae articulate at more than one surface. This paper proposes a method to estimate 3D motion between two avian vertebrae, by bones surface reconstruction and contact modeling. The neck of birds was selected as a case study because it is a functionally highly versatile structure combining dexterity and strength. As such, it has great potential to serve as a source for bioinspired design, for robotic manipulators for instance. First, 3D models of the vertebrae are obtained by computed tomography (CT). Next, joint surfaces of contact are approximated with polynomial surfaces, and a system of equations derived from contact modeling between surfaces is established. A constrained optimization problem is defined in order to find the best position of the vertebrae for a set of given orientations in space. As a result, the possible intervertebral range of motion is estimated.
\end{abstract}

\section{KEYWORDS}

Bird vertebrae, Articular surfaces, Contact modeling, Kinematics

\section{Introduction}

To get inspiration from nature in robotics, two factors have to be associated: a biological model corresponding to the technological requirements and a deep understanding of its mechanical features. In birds, the neck is thought to have become the equivalent to an arm because it is used for feeding, cleaning, and many other tasks. Due to this functional versatility, the avian neck offers a number of interesting performances that are of high interest for robotic manipulators (Abourachid and Wenger 2019). However, their mechanical features need to be better understood. Available data in the literature are mainly composed of measurements to evaluate the cervical joint range of motion (Robert E. Kambic and Pierce 2017; Cobley et al. 2013), but these data do not allow for reconstructing the motion between vertebrae. Such a reconstruction is 


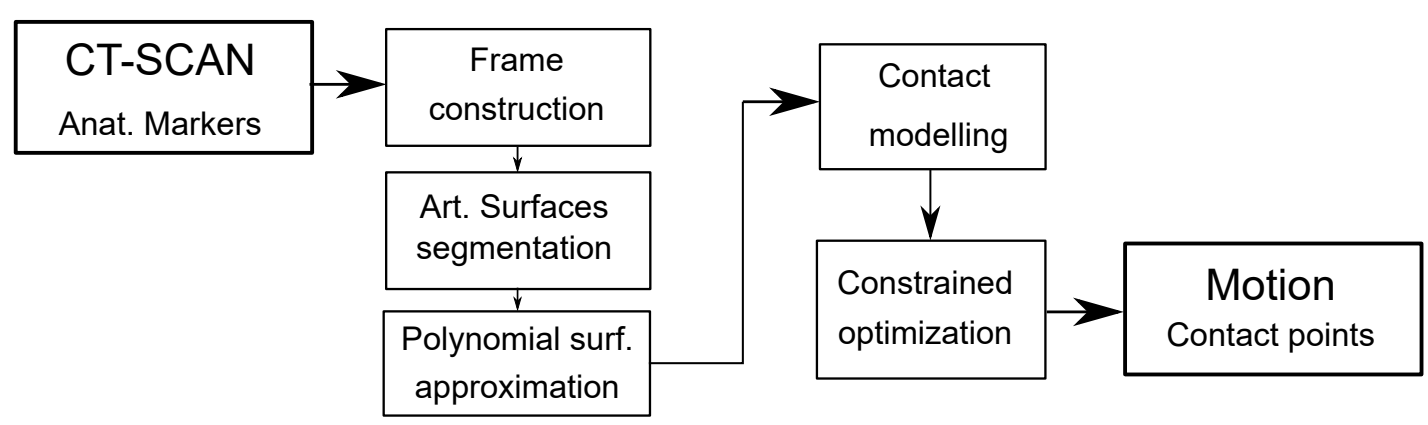

Figure 1.: Workflow scheme of the proposed method

a necessary step to build a bio-inspired robotic model with suitable kinematic joints and may bring a better understanding of the neck's functional behavior.

To reach this goal, the bird neck can be modeled as a multi-body system linked by joints. The bird's neck modeling can be complex. It includes kinematic and dynamic models. The kinematic model focuses on the pure motion between bodies regardless of the actuation forces needed to move those bodies while the dynamic modeling considers both motion and forces of a system. We decided to focus here only on the kinematic modeling of the bird neck, the definition of the motion between two adjacent vertebrae. The motion of bird necks is difficult to observe and quantify. Indeed, as the neck is covered with feathers, its real motion is most often hidden. Moreover, in vivo measurements are very difficult to conduct.

Biological joint motions have been extensively studied for clinical purposes in human knees (Siegler et al. 2018). Two main approaches to bone interaction and joint modeling can be distinguished. The first one is the study of the contact itself (location and stress) for different joint positions obtained from physical measurements (Islam et al. 2015: Johnson et al. 2014, Gustafson et al. [date unknown]), and is based on finite elements (FE) methods and elastic models. The second approach is the spatial relationship between articular surfaces during a motion (Siegler et al. 2018; Parenti-Castelli et al. 2004: Corazza et al. 2005: Ottoboni et al. 2010). In contrast to the knee, the cervical vertebral column is composed of several small bones. It is, thus, difficult to have in-vivo measurements, and to pursue the first approach. Moreover, in contrast to mammals that have intervertebral discs, the vertebrae in birds articulate via very thin synovial joints that act more as a fluid (Böhmer et al. 2019). Thus, a deformable body approach is meaningless for contacts between avian vertebrae, displaying a unique intervertebral articulation morphology: the bones articulate via heterocoelous (saddleshaped) surfaces (Boas 1929). This morphology allows for both mobility and stability, and makes the motion between two vertebrae more traceable than other intervertebral articulation morphologies, such as acoelous joints (flat surfaces; e.g., in mammals).

This paper proposes a method that uses computed tomography (CT) scans of vertebrae in order to reconstruct the motion between two avian vertebrae on the sole basis of a kinematic point of view. First, the articular surfaces are extracted from the CT-scans and approximated with polynomial surfaces (Ottoboni et al. 2010; Boyd et al. (1999). Second, a model of contact between surfaces is derived. Then, for a given orientation between two vertebrae, a numerical solution of the contact equations is computed. As a result, the motion between two vertebrae can be computed. The proposed method is summarized in figure 1. Finally, results are presented for the motion between two vertebrae of the black woodpecker (Dryocopus martius). 


\section{Materials and Methods}

In general, a vertebra is composed of two structural elements: the ventral centrum (vertebral body) and the dorsal neural arch. Together they form a cavity for the spinal cord passage. The neural arch bears cranial and caudal articular processes the zygapophyses. Two vertebrae articulate via their centra and zygapophyses, in combination with constraints imposed by soft tissue. Those contact areas define and limit the motion between vertebrae and have different shapes for each vertebra. The two criteria that we use in the present study to limit the intervertebral motion are bone collision and osteological disarticulation (Krings et al. 2017; Arnold et al. 2014 ; Nyakatura et al. 2015). It is likely that the limits found by our algorithm are not penalizing for the real movements which will be in practice more reduced.

\subsection{Acquisition of $3 D$ models}

The vertebrae were scanned by micro-computer tomography. The 3D models of the vertebrae were reconstructed from the $\mu \mathrm{CT}$ scans (voxel size: $0.054 \mathrm{~mm}$ ) using the Avizo software (Terray et al. 2019). The vertebrae considered in this paper are the $V_{5}$ and $V_{6}$ vertebrae of a black woodpecker (total number of vertebrae in the neck: 11). We selected the two vertebrae from the intermediate region of the neck because the vertebrae from both the cranial and caudal region generally display a highly specialized morphology.

In order to work on the relative motion between two vertebrae, a frame attached to each body $\mathcal{B}$ has to be considered. To do so, three anatomical markers are considered. The first marker $S_{0}$ is positioned at the center of the caudal centra, the second one $S_{1}$ at the center of the cranial centra and the last one $S_{2}$ at the most dorsal point of the neural spine. A coordinate system is defined as follows (see figure 2 and 3 ) :

- The origin of the frame is defined as the middle point between $S_{1}$ and $S_{0}$

- The cranio-caudal $x$-axis is defined by the vector $\overrightarrow{S_{1} S_{0}}$

- The medio-lateral $z$-axis is normal to the plane $\left(S_{0} S_{1} S_{2}\right)$ and following the righthand-rule convention.

- The ventro-dorsal $y$-axis is normal to both $x$ and $z$-axis, following the right-hand-rule convention.

\subsection{Acquisition of physical articular limits}

The motion considered in this paper is the spatial motion between the fifth and sixth vertebra of a black woodpecker (namely $V_{5}$ and $V_{6}$ ). For this specimen, the physiological ex-vivo rotation limits have been obtained from X-ray measurements (see figure 4). After having removed all feathers and other soft-tissue, the dead body was positioned on its lateral side with the first cranial thoracic vertebrae fixed to the horizontal working bench. We inserted a plumb bead into the right and the left ear canal. In the X-ray image, both plumb beads are superimposed which enabled us to prevent that the head-neck system is distorted. Next, the neck was subjected to maximal flexion in dorsal and ventral direction. The dead body was then positioned on its dorsal side with the first cranial thoracic vertebrae fixed to the working bench in order to subject the neck to maximal lateral rotation. At each of these configurations, the excursion of a single degree of freedom was maximized which allowed us to record the maximal intervertebral angle without damaging muscles or ligaments. Although this treatment 


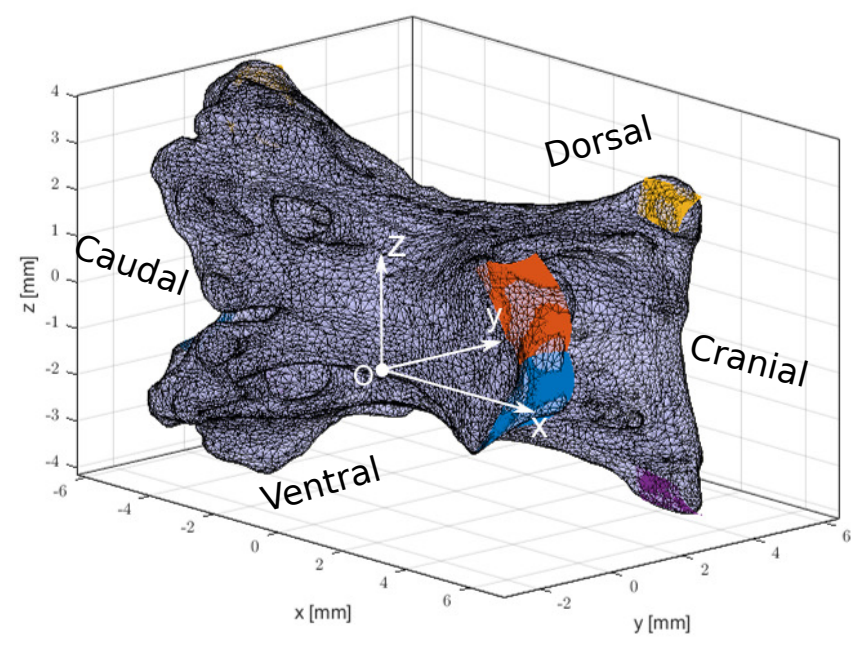

Figure 2.: Model of a scanned bird vertebrae with approximated joint contact surfaces displayed in color (four on the cranial side and four on the caudal side).

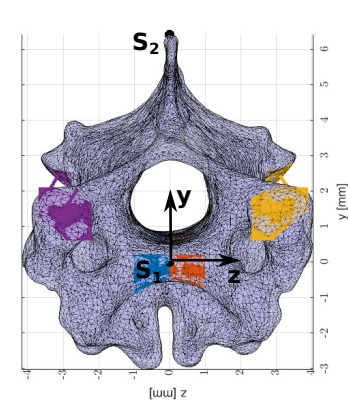

(a)

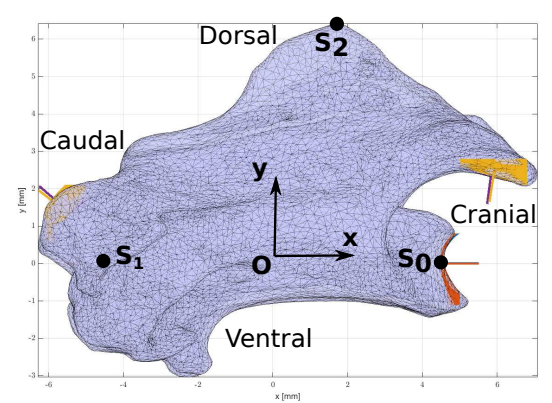

(b)

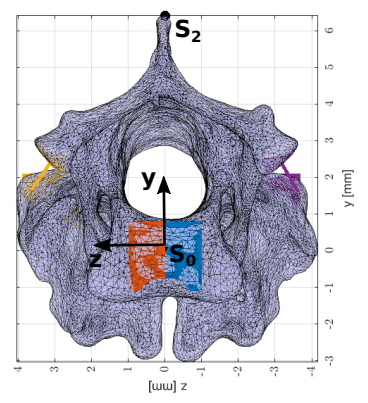

(c)

Figure 3.: Different view of a bird vertebrae scan : (a) front view of the cranial side, (b) side view, (c) front view of the caudal side. The colored parts corresponds to the eight contact areas used in contact modeling (four on the cranial side and four on the caudal side).

creates an artificial situation, it yields important insights into the movements possible during large head movements (Krings et al. 2017).

\subsection{Contact modeling Method}

A completely free motion between two bodies has six degrees of freedom (dof) described as three translations and three rotations. The observation of the real motion between two vertebrae suggests that it has only two dof, associated with motions in the frontal and sagittal plane, respectively. Indeed, the rotation about the cranio-caudal $x$-axis is fully constrained by the contacts. This mechanical blockage prevents the spinal cord, that goes through the vertebrae, to be torned. At first glance, the two above mentioned motions can be defined by one rotation about the ventro-dorsal $y$-axis and another rotation about the medio-lateral z-axis. Note that since the motion between two bird neck vertebrae is complex, the rotations described above are likely to be 


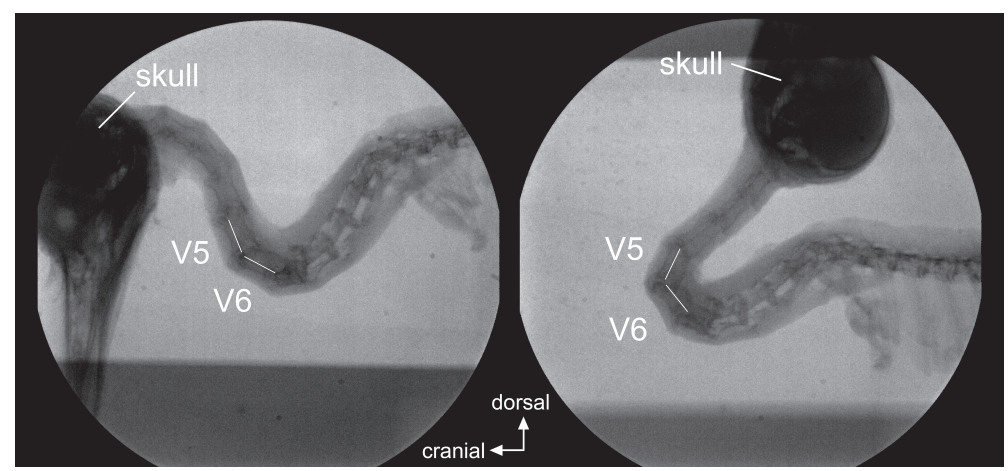

Figure 4.: X-ray of a black woodpecker showing the neck in ex vivo neutral position (left) and maximal dorsal flexion (right). The cervical vertebrae five (V5) and six (V6) are highlighted with an axial line to visualize the angle between them.

non pure rotations, i.e. a coupled translation is involved in the motion plane. Such coupled motions exist when the center of rotation moves in the motion plane, which is the case in the knee joint (Parenti-Castelli et al. 2004). An estimate of the motion can be defined thanks to contact modeling, by defining a certain number of contact points that constraint the two vertebrae to be positioned in a precise way. Since one contact point between two surfaces removes one dof and since there are only two dof between two adjacent vertebrae, four contact points defined on four surfaces can produce the expected mobility. Now, a contact between two surfaces can be described with four contact equations: two equations stipulating that the surfaces share at least one common point and two equations imposing that the surfaces share the same normal at their common point. Together, these four equations imply that the surfaces touch on a unique contact point. On the other hand, a contact between two surfaces introduces three unknowns, the three Cartesian coordinates of the contact point to be determined. Since four contact points between the two vertebrae are considered, we come up with 16 equations for 18 unknowns (12 coordinates of contact points and 6 dof), resulting in two supplementary undetermined unknowns. Since the relative motion of two adjacent vertebrae is assumed to rely on only two rotations, considering those two rotations as parameters makes it possible to define a fully determined problem.

\subsection{Joint Surfaces segmentation and approximation}

Once the coordinates attached to each vertebrae are defined, one need to define the joint surfaces areas. For each vertebra, eight distinct contact surfaces areas are defined: two areas are on the centrum of the vertebrae, and two areas on the zygapophyses (on both cranial and caudal sides, see figs 2 and 3), keeping in mind that the 4 cranial surfaces articulate with the 4 caudal surfaces of previous vertebra, and the 4 caudal surfaces articulate with the 4 cranial surfaces of next vertebra. As the bone scans are very accurate and the contact surfaces easy to identify and separate from the rest of the bone surface, the nodes corresponding to the surfaces are selected by hand and isolated from the rest of the cloud of dots with the help of a CAD software (3DExperience).

In order to derive contact equations, real surfaces are approximated with the fit 


\begin{tabular}{c|c|c|c|c} 
& \multicolumn{2}{|c|}{ error centrum } & \multicolumn{2}{c}{ error zygapophyses } \\
\hline & $\mathrm{n}=2$ & $\mathrm{n}=3$ & $\mathrm{n}=1$ & $\mathrm{n}=2$ \\
\hline$V_{5}$ & 0.066 & 0.038 & 0.11 & 0.019 \\
\hline$V_{6}$ & 0.057 & 0.035 & 0.077 & 0.018
\end{tabular}

Table 1.: Mean error (in $\mathrm{mm}$ ) for the two vertebrae for different orders of polynomial surfaces.

function of Matlab, which generates polynomial surfaces defined as in equation (1).

$$
S: f(u, v, w)=\sum_{h=0}^{n} \sum_{l=0}^{n} p_{h l} u^{h} v^{l}-w=0
$$

With $(u, v, w)$ the coordinates in the surface-centered frame, and $n$ the order of the polynomial surface. The main axes $w$ or depth of each surface needs to be chosen wisely. For the centrum surfaces (i.e the red and blue surfaces in figs 2 and 3 ), the general $x$-axis calculated before is chosen as the height axis $w$ of the polynomial surfaces. Indeed, the $S_{0}$ and $S_{1}$ points defining the $x$-axis are directly chosen from the center of the vertebral body surfaces. Accordingly, the polynomial equation is of form (2).

$$
S_{c}: f_{c}(x, y, z)=\sum_{h=0}^{n} \sum_{l=0}^{n} p_{h l} y^{h} z^{l}-x=0
$$

For the zygapophyses, however, the direction of the normal axis of the surface is not straightforward. Since the zygapophysis surfaces are mainly flat (contrary to the centrum surfaces), the normal axis is chosen by computing the least square plane (i.e the fit function with a first order polynomial, $n=1$ ). The axis found is then used as the normal axis $w$ to generate polynomial surfaces. The polynomial function obtained in the local frame $(u, v, w)$ of the zygapophysis is then transformed in the global frame $(x, y, z)$ of the vertebrae, resulting in an equation of form (3).

$$
S_{z}: f_{z}(x, y, z)=\sum_{h=0}^{n} \sum_{l=0}^{n} \sum_{m=0}^{n} p_{h l m} x^{h} y^{l} z^{m}=0
$$

The order of the polynomial is chosen such that the mean error, i.e the normal distance between the computed surfaces and the real points of the CT scans is less than the voxel size of the CT scan (i.e $0.054 \mathrm{~mm}$ ). Table 1 shows that at least a third order is needed for the centrum surfaces approximation, whereas a second order is sufficient for the zygapophysis. The reason for this is that the zygapophysis are quite flat, which makes determination of their normal axis $w$ more accurate.

Polynomials coefficients are not detailed but are given directly by the fit function for equation (2) and also depends on the zygapophysis local frame for equation (3). 


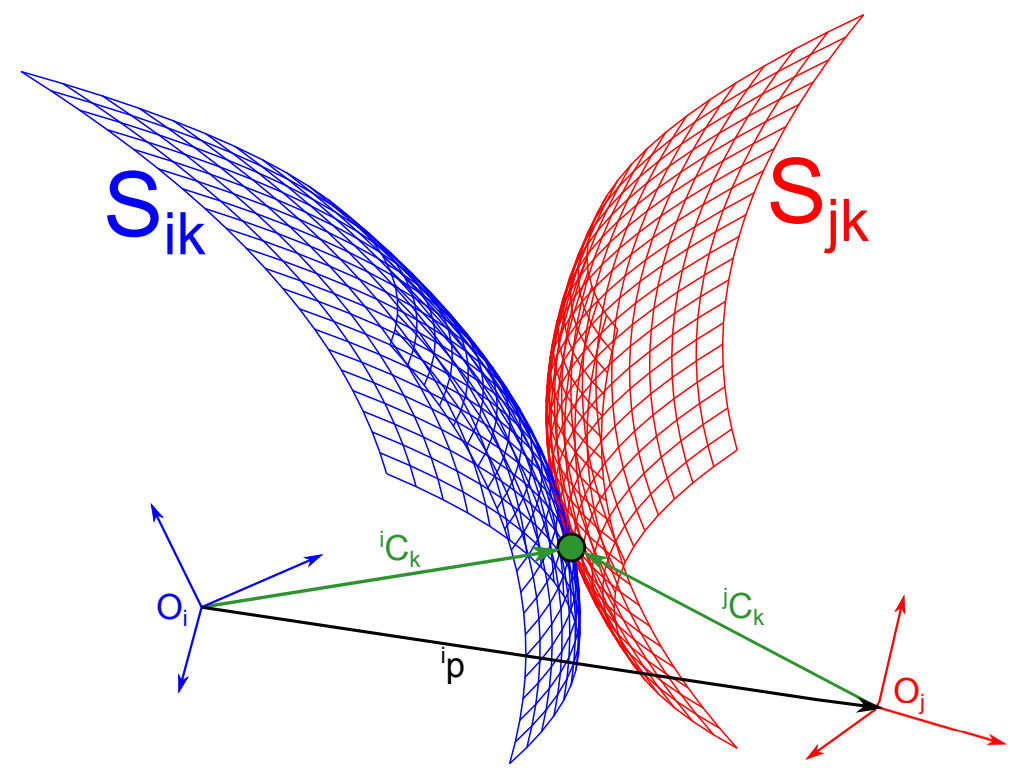

Figure 5.: Representation of two surfaces $S_{i k}$ and $S_{j k}$ in contact at point $C_{k}$.

\section{Numerical computation}

\subsection{Contact modeling}

The $i^{\text {th }}$ vertebra articulates its caudal side with the cranial side of the $j^{\text {th }}$ vertebra. Let us define two surfaces $S i_{k}$ and $S j_{k}$ attached to vertebra bodies $\mathcal{B}_{i}$ and $\mathcal{B}_{j}$, respectively and written in frames $\mathcal{R}_{i}$ and $\mathcal{R}_{j}$, respectively (see figure 5). The above mentioned bodies are adjacent in the neck chain, $j=i+1$, but for a matter of compactness, notation $j$ is kept. Index $k$ stands for the contact surface number: $k=1,2,3,4$. The surfaces equations are:

$$
\begin{aligned}
S i_{k}\left({ }^{i} x,{ }^{i} y,{ }^{i} z\right) & =0 \\
S j_{k}\left({ }^{j} x,{ }^{j} y,{ }^{j} z\right) & =0
\end{aligned}
$$

where $\left({ }^{i} x,{ }^{i} y,{ }^{i} z\right)\left(\right.$ resp. $\left.\left({ }^{j} x,{ }^{j} y,{ }^{j} z\right)\right)$ are the coordinates of a point in frame $\mathcal{R}_{i}$ (resp. in frame $\mathcal{R}_{j}$ ).

The surface expressions depend on the contact surface number k. For $k=\{1,2\}$, the surface is a centra and equation (2) is taken, whereas for $k=\{3,4\}$, the surface is a zygapophysis and we take equation (3).

Let us also define the gradients of the two surfaces expressed in their frame:

$$
\begin{aligned}
& { }^{i} \nabla S i_{k}=\left(\frac{\partial S i_{k}}{\partial^{i} x}, \frac{\partial S i_{k}}{\partial^{i} y}, \frac{\partial S i_{k}}{\partial^{i} z}\right)^{\top} \\
& { }^{j} \nabla S j_{k}=\left(\frac{\partial S j_{k}}{\partial^{j} x}, \frac{\partial S j_{k}}{\partial^{j} y}, \frac{\partial S j_{k}}{\partial^{j} z}\right)^{\top} \\
& k=1,2,3,4
\end{aligned}
$$


Two surfaces are in contact if and only if they satisfy the following conditions: (i) they have a common contact point $C_{k}$ and $(i i)$ the two surfaces have a common normal in the contact point (Siegler et al. 2018, Parenti-Castelli et al. 2004, Corazza et al. 2005: Ottoboni et al. 2010). These two conditions can be expressed with four equations, namely $C_{k}$ satisfies equation (4) and (5) and the tangent plane of the two surfaces is the same at contact point $C_{k}$, i.e by taking appropriate coordinates of (6) and (7) (Di Gregorio and Parenti-Castelli 2003):

$$
\left\{\begin{array}{l}
S_{i k}\left({ }^{i} \mathbf{C}_{k}\right)=0 \\
S_{j k}\left({ }^{j} \mathbf{C}_{k}\right)=0 \\
{ }^{i} \nabla S_{i k} x^{i} \nabla S_{j k} z-{ }^{i} \nabla S_{j k} x^{i} \nabla S_{i k} z=0 \\
{ }^{i} \nabla S_{i k} y^{i} \nabla S_{j k} z-{ }^{i} \nabla S_{j k} y^{i} \nabla S_{i k} z=0
\end{array}\right.
$$

Note that the above equations must be handled in the same frame. Let ${ }^{i} \mathbf{p}=$ $\left[t_{x}, t_{y}, t_{z}\right]^{\top}$ be the position vector of the center of frame $\mathcal{R}_{j}$ in $\mathcal{R}_{i}$. The relation linking the coordinates of ${ }^{i} \mathbf{C}_{k}$ and ${ }^{j} \mathbf{C}_{k}$ in frame $\mathcal{R}_{i}$ and $\mathcal{R}_{j}$, respectively, can be written as follows (Khalil and Dombre 2004):

$$
{ }^{i} \mathbf{C}_{k}={ }^{i} \mathbf{R}_{j}{ }^{j} \mathbf{C}_{\mathbf{k}}+{ }^{i} \mathbf{p}
$$

where ${ }^{i} \mathbf{R}_{j}$ is the rotation matrix that transforms an element in $\mathcal{R}_{i}$ into an element in $\mathcal{R}_{j}$, described with extrinsic Euler angles $[\alpha, \beta, \gamma]^{\top}$, defined as rotation around $\mathrm{x}, \mathrm{y}$ and $\mathrm{z}$ axis of the $i^{\text {th }}$ vertebra:

$$
\begin{aligned}
{ }^{i} \mathbf{R}_{j} & =\operatorname{Rot}(x, \alpha) \operatorname{Rot}(y, \beta) \operatorname{Rot}(z, \gamma) \\
{ }^{i} \mathbf{R}_{j} & =\left[\begin{array}{ccc}
1 & 0 & 0 \\
0 & C \alpha & S \alpha \\
0 & -S \alpha & C \alpha
\end{array}\right]\left[\begin{array}{ccc}
C \beta & 0 & -S \beta \\
0 & 1 & 0 \\
S \beta & 0 & C \beta
\end{array}\right]\left[\begin{array}{ccc}
C \gamma & S \gamma & 0 \\
-S \gamma & C \gamma & 0 \\
0 & 0 & 1
\end{array}\right]
\end{aligned}
$$

where $\mathrm{C}$ and $\mathrm{S}$ stand for the cosine and sine function, respectively.

Note that relation $(10)$ stands for points. For vectors, the last term ${ }^{i} \mathbf{p}$ must be removed (Khalil and Dombre 2004).

As observed before, a system of four equations at each contact point is then obtained, and a contact point introduces three unknowns (the three position coordinates of $C_{k}$ ). Since frames $\mathcal{R}_{i}$ and $\mathcal{R}_{j}$ are linked by equation $(10)$, system $(9)$ also depends on the position vector ${ }^{\mathbf{i}} \mathbf{p}$ and the rotation matrix ${ }^{i} \mathbf{R}_{j}$.

\subsection{Definition of the optimal problem for numerical resolution}

The 2 vertebrae have 2 degrees of mobility with respect to each other. These mobilities are parameterised by 2 variables $\beta$ and $\gamma$ which are rotations about the $\mathrm{y}$ and $\mathrm{z}$ axes. We therefore want to write the contacts between the vertebrae for a given set of $\beta$ and $\gamma$ values within the limits of the expected motion of the vertebrae. As shown before, the motion between two adjacent vertebrae of the bird neck can be defined with four contact points that constraint the two vertebrae to be positioned in a precise way: two contact points on the centrum (one on each side of the sagittal plane of the vertebrae, namely plane $(x O y)$ ), and one contact point on each zygapophysis. Since each contact point involves four equations, there are 16 contact equations to be solved together. 
The unknows are the coordinates of the 4 points of contact resulting in 12 unknows and the relative position/orientation of the two vertebrae. Since the rotation $\beta$ and $\gamma$ about $\mathrm{y}$ and $\mathrm{z}$ axes are imposed, 4 relatives position and orientation has to be defined. $\left[t_{x}, t_{y}, t_{z}\right]$ and the rotation $\alpha$ about the $\mathrm{x}$ axis.

These two angle variables are discretized and for each value of $\beta$ and $\gamma$, we can determine the corresponding position $\left[t_{x}, t_{y}, t_{z}\right]$ and rotation angle $\alpha$ satisfying all the contact equations (i.e: each surface $S i_{k}$ of body $i$ is in contact with its corresponding surface $S j_{k}$ of body $j$ at point $C_{k}$ ). A full-rank system of equations is then obtained. However, this system of equations is non-linear and can admit a solution or not and jumps in solutions can occur for small angular variations. This is due to the difficulty of simultaneously adjusting contact points over several areas. In reality, the surfaces that are put in contact are not rigid bodies but there are a sinovial joint between the surfaces. To model this, an offset modeling sinovial joint thickness is introduced in the equation. The contact equations become :

$$
\left\{\begin{array}{l}
S_{i k}\left({ }^{i} \mathbf{C}_{k}\right)=d_{i k} \\
S_{j k}\left({ }^{j} \mathbf{C}_{k}\right)=d_{j k} \\
{ }^{i} \nabla S_{i k} x^{i} \nabla S_{j k} z-{ }^{i} \nabla S_{j k} x^{i} \nabla S_{i k} z=0 \\
{ }^{i} \nabla S_{i k} y^{i} \nabla S_{j k} z-{ }^{i} \nabla S_{j k} y^{i} \nabla S_{i k} z=0
\end{array}\right.
$$

Note that the equations describing the condition on the normal to the surface are not modified. The value of $d_{i k}$ and $d_{j k}$ are unknown and can vary along the trajectory (i.e it depends on $\beta$ and $\gamma$ ), but is allowed to vary between $\pm 0.05 \mathrm{~mm}$ only.

The system of equations, is now under-determined as eight new parameters $\mathbf{d}_{\mathbf{i}}=$ $\left[d_{i 1}, d_{i 2}, d_{i 3}, d_{i 4}\right]^{\top}$ and $\mathbf{d}_{\mathbf{j}}=\left[d_{j_{1}}, d_{j_{2}}, d_{j_{3}}, d_{j_{4}}\right]^{\top}$ have been introduced. This system is solved as an optimal problem under constraints defined as follows:

$$
\min _{\mathbf{q}} f(\mathbf{q}) \text { such that }\left\{\begin{aligned}
& \mathbf{c}(\mathbf{q}) \leq 0 \\
& \mathbf{l}_{b} \leq \mathbf{q} \leq \mathbf{u}_{b}
\end{aligned}\right.
$$

where $\mathbf{q}=\left[\mathbf{d}_{\mathbf{i}}, \mathbf{d}_{\mathbf{j}},{ }^{i} \mathbf{C}_{1},{ }^{i} \mathbf{C}_{2},{ }^{i} \mathbf{C}_{3},{ }^{i} \mathbf{C}_{4}, t_{x}, t_{y}, t_{z}, \alpha\right]^{\top}$ is the optimization vector. $f$ is the objective function. Since we want to have a solution to equation $(12)$, the objective function to minimize is chosen as the least square error of the sum of contact equations:

$$
\begin{aligned}
& f(\mathbf{q})=\sum_{4 k}\left[\left(d_{i k}+S_{i k}\left({ }^{i} \mathbf{C}_{k}\right)\right)^{2}+\left(d_{j k}+S_{j k}\left({ }^{j} \mathbf{C}_{k}\right)\right)^{2}\right. \\
& +\left({ }^{i} \nabla S_{i k} x{ }^{i} \nabla S_{j k} z-{ }^{i} \nabla S_{j k} x^{i} \nabla S_{i k} z\right)^{2} \\
& \left.+\left({ }^{i} \nabla S_{i k} y^{i} \nabla S_{j k} z-{ }^{i} \nabla S_{j k} y^{i} \nabla S_{i k} z\right)^{2}\right]
\end{aligned}
$$

c is the vector of nonlinear equality constraints, $\mathbf{l}_{b}$ (resp. $\mathbf{u}_{b}$ ) the vector of lower bounds (resp. upper bounds) of the optimization vector. As the surface equations (4) and (5) do not take into account physical bounds of the articular surfaces, the two vector $\mathbf{l}_{b}$ and $\mathbf{u}_{b}$ and vector $\mathbf{c}$ impose the contact points to be inside a prism that includes the entire articular surface, and respectively for each contact point, and for surface (4) and (5). 

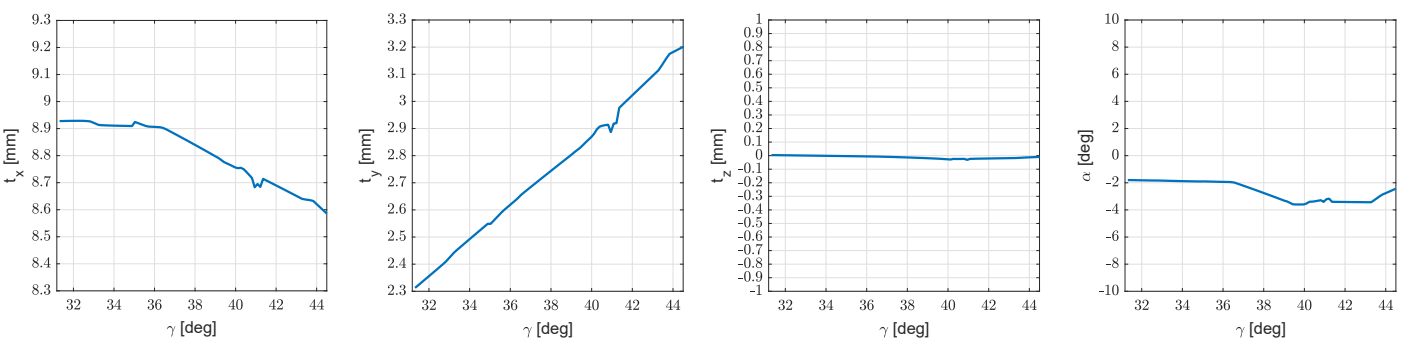

Figure 6.: Evolution of the position between the two vertebrae for $\gamma \in\left[31.2^{\circ}, 44.5^{\circ}\right]$ and $\beta=0^{\circ}$.

To solve this problem, we used the Multistart function of Matlab which is a function to help finding the global optimal problem under constraints. This function attempts to find multiple local solutions to a problem by starting from various points. It uses the fmincon function with the sqp algorithm that ensures the respect of the non-linear constraints and bounds at each iteration.

\section{Results and discussion}

\subsection{Motion modeling of two vertebraes}

As a combined motion involving two rotations about $y$ and $z$ is difficult to represent, the two possible rotations are shown separately. Figure 6 shows the evolution of frame coordinates with respect to $\gamma$ for $\beta=0^{\circ}$, namely translation $t_{x}, t_{y}$ and $t_{z}$ between frame $\mathcal{R}_{5}$ and $\mathcal{R}_{6}$, and rotation $\alpha$ about $x$. Rotation in sagittal plane $\gamma$ has been discretized between the bounds obtained on X-rays measurements (figure 4), so $\gamma \in\left[31.2^{\circ}, 44.5^{\circ}\right]$. It can be seen that the algorithm converges for all the considered values of $\gamma$, meaning that for each discretized value of $\gamma$ a solution minimizing $f$ and satisfying the constraints equation is found. To reduce local minima effects, fmincon is run several times with different starting points using the global optimization toolbox and multistart function. The largest amplitude of motion is observed for the translation $t_{y}$ (see figure 6). The $t_{z}$ translation is close to zero and does not vary much, which was expected for a motion in the sagittal plane. We could also expect that $\alpha=0$ as the physical observation, nonetheless we see that $\alpha$ rotation vary between $-4^{\circ}$ and $-2^{\circ}$. Two types of errors can be observed, the offset error of the mean value, that comes from the frame construction of the vertebrae which is not exact and variation errors due to the surface approximation.

A rotation is also performed about $y$. Figure 7 shows the evolution of frame coordinates with respect to $\beta$ for a fixed value of $\gamma=37.9^{\circ}$, the mean value between $\gamma_{\min }$ and $\gamma_{\max }$. The angle $\beta$ was discretized between the bounds obtained on X-rays measurements, so $\beta \in\left[-15^{\circ}, 15^{\circ}\right]$. As expected, the largest variation is for the $t_{z}$ value. The $t_{x}$ and $t_{y}$ values vary less, but are less smooth than for the motion around $z$. This can be due to the numerical computation, which does not link the solutions for different values of $\beta$, meaning that different local minimums can be found from one value to an other. Since the motion is a rotation about $y$, we can see that the evolution of $t_{x}, t_{y}$ and $\alpha$ is close to symmetric, as it could be expected.

A video that compiles motion of 6 and 7 is available at http://videos. 

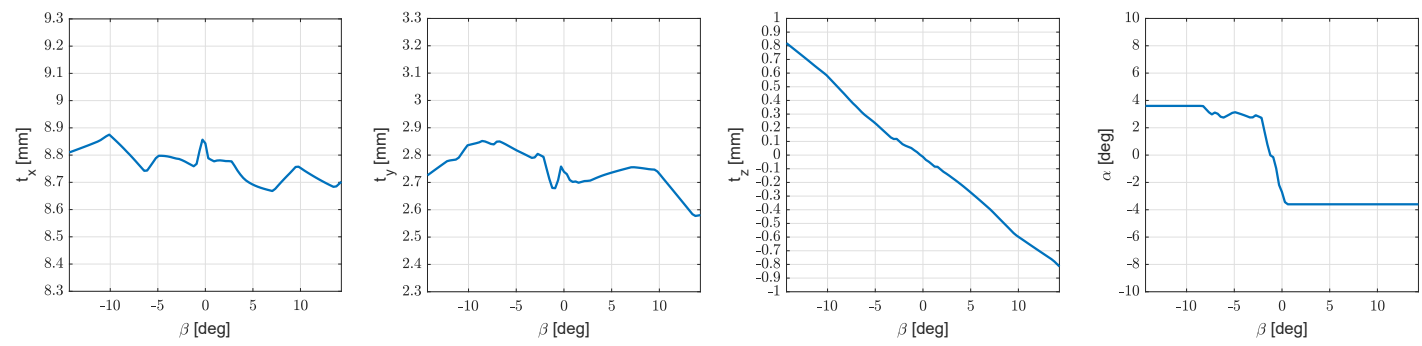

Figure 7.: Evolution of the position between the two vertebrae for $\beta \in\left[-15^{\circ}, 15^{\circ}\right]$ and $\gamma=37.9^{\circ}$.

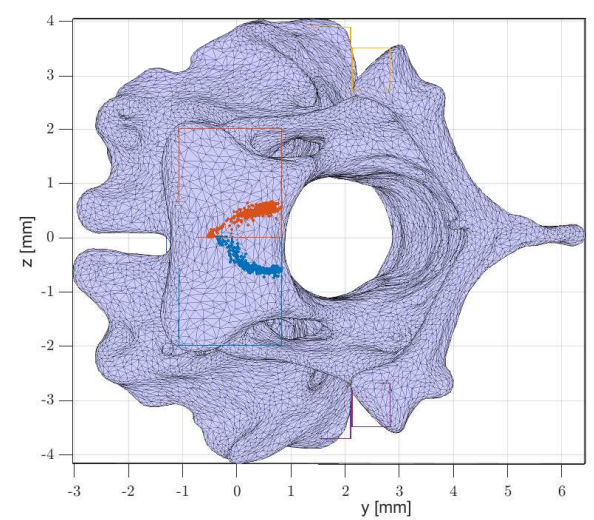

(a)

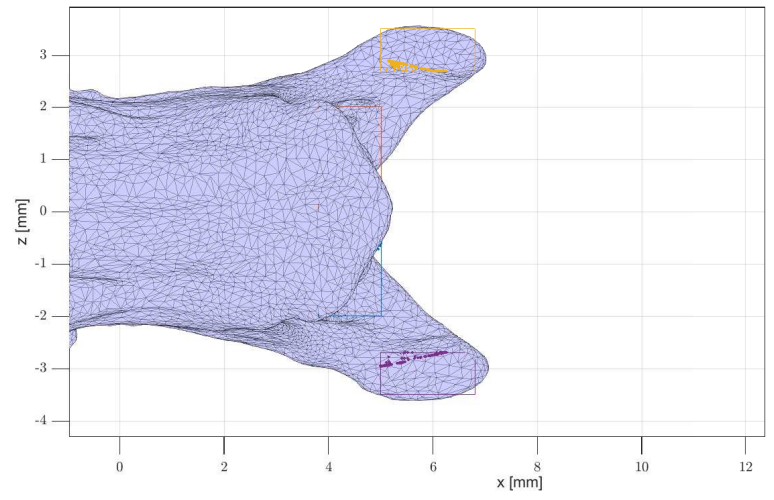

(b)

Figure 8.: Evolution of contact point location on vertebra 5 for the complete possible motion for central articular surface (a) and zygapophyses (b).

univ-nantes.fr/medias/2020/05/f7d5863c22b38c8322d5377a376872f7.mp4. The observation based on this animation validates the methodology, as the motion between vertebrae is smooth and the contact surfaces are respected. For all sets of possible orientations $[\beta, \gamma]$, the coordinates of the contact points can be obtained (see figure 8). It can be seen that the position of contact points are located around a small zone of the full articular surface.

\subsection{Comparison between measured and calculated flexion limits}

We found by observing the value of the optimization function $f$ an interesting secondary result (figure 9). We tried in the sagittal plane to discretize the $\gamma$ angle for a larger interval that the one measured with X-rays. We found out that the value of $f$ is increasing exponentially once the angle $\gamma$ reaches a specific value. This value for dorsal flexion is close to the physical articular limit $\gamma_{\max }$, but for the ventral flexion, the error is not increasing beyond the $\gamma_{\min }$ value. This result is in accordance with real observation on vertebrae. In fact, for the dorsal flexion, we see that the articular limit is a physical limit of contact between bones, that cannot be exceeded because the two vertebraes are in contact on their neural crest side. This contact is not taken into account in this model but corresponds to a limit of contact on the zygapophysis surfaces. On the ventral side, however, the measured limit is due to the action of mus- 


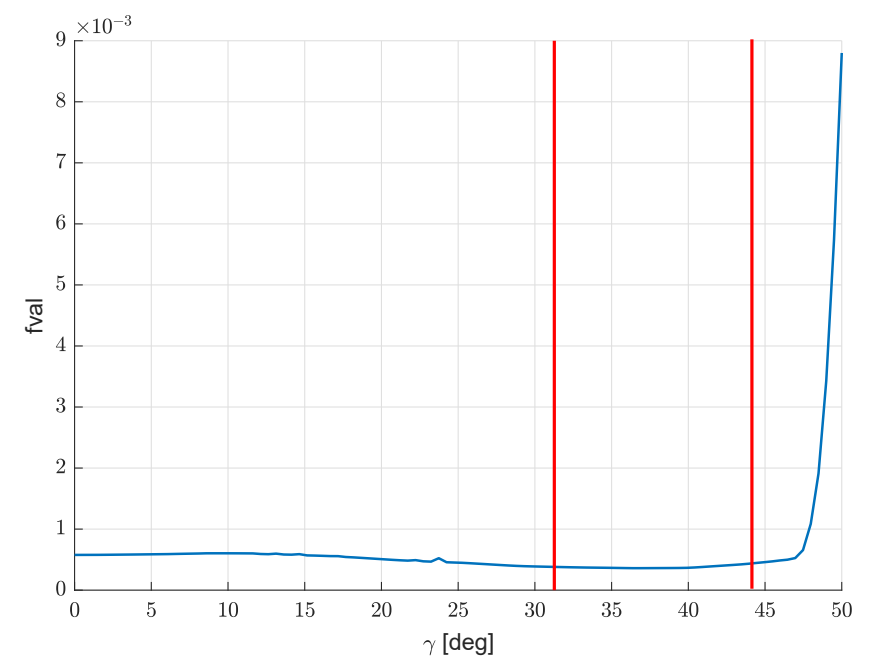

Figure 9.: Value of $f(q)$ for $\gamma$. The measured limits are shown in vertical red lines.

cles and tendons, which are mainly located on the dorsal side of the bird neck and could participate to a restriction of the ventral flexion (Böhmer et al. 2019). Since our method is only based on contact modeling of bones, the interaction with tendons and muscles is not taken into account, which makes the possible range of motion in ventral flexion greater that the one measured.

\section{Conclusion}

Real observation and in-vivo measurements on animals are difficult to obtain for several reasons. In this paper, a new method to compute motions between two bones has been developed. Geometric polynomial surfaces have been derived on the basis of precise and real CT-scans of vertebrae. Upon establishing contact equations between articular surfaces and applying a constraint optimization algorithm, the motion between vertebrae can be reconstructed. The obtained motions are an estimation of the real ones and needs to be further compared with real measurements as much as possible. Up to now, the obtained motions are validated on a qualitative aspect only. In contrast to some other intervertebral articulation morphologies, the heterocoelous vertebrae of birds offer the advantage that the intervertebral motion is more traceable. It has been shown that the real motion between vertebrae is complex, and recent results show that depending on the considered vertebrae, the motion can be more complex than a pure rotation. The assumption of no torsion between two bird vertebrae has also been verified. The method can be applied to a complete set of vertebrae, describing each relative motion between two of them. By reconstructing the whole motion, it is possible to extend the results to at least a full bird neck model. It would then be possible to partially validate the method, as the full motion of the neck is easier to observe than the inter-vertebrae motion.

Future work will focus on the kinematic modeling of such an articulation by defining an appropriate spatial mechanism with kinematic chain that fits best with the relative motion between two vertebrae. Following the same method, a 3D model considering several vertebrae and the whole neck, respectively, will be eventually created and 
compared with the results obtained from the ex-vivo range of motion analysis. This method can be applied to any joint that has its motion defined by contact surfaces. It could be applied to other biological joints in order to reconstruct motions from CTscan of bones, and be useful on clinical aspects. The method could be used for orthesis or re-education device design, or to study the effect of articular surfaces malformation on the joint motion.

\section{Acknowledgment}

This work was conducted with the support of the French National Research Agency (AVINECK Project ANR-16-CE33-0025).

\section{References}

Abourachid A, Wenger P. 2019. Avineck, the neck of the bird, an arm for the robots. In: 44ème congrès de la Société de Biomécanique; Oct; Poitiers, France.

Arnold P, Fischer MS, Nyakatura JA. 2014. Soft tissue influence on ex vivo mobility in the hip of iguana: comparison with in vivo movement and its bearing on joint motion of fossil sprawling tetrapods. Journal of anatomy. 225(1):31-41.

Boas JEV. 1929. Biologisch-anatomische studien über den hals der vögel. vol. 9. AF Høst \& $\mathrm{s} \varnothing \mathrm{n}$.

Boyd S, Ronsky J, Lichti D, Salkauskas D, Chapman M. 1999. Joint surface modeling with thin-plate splines.

Böhmer C, Prevoteau J, Duriez O, Abourachid A. 2019. Gulper, ripper and scrapper: anatomy of the neck in three species of vultures. Journal of Anatomy (2019).

Cobley MJ, Rayfield EJ, Barrett PM. 2013. Inter-vertebral flexibility of the ostrich neck: implications for estimating sauropod neck flexibility. PLoS One. 8(8).

Corazza F, Stagni R, Castelli VP, Leardini A. 2005. Articular contact at the tibiotalar joint in passive flexion. Journal of biomechanics. 38(6):1205-1212.

Di Gregorio R, Parenti-Castelli V. 2003. A spatial mechanism with higher pairs for modelling the human knee joint. J Biomech Eng. 125(2):232-237.

Gustafson JA, Elias JJ, Debski RE, Farrokhi S. [date unknown]. Development and validation of a kinematically-driven discrete element model of the patellofemoral joint.

Islam K, Duke K, Mustafy T, Adeeb SM, Ronsky JL, El-Rich M. 2015. A geometric approach to study the contact mechanisms in the patellofemoral joint of normal versus patellofemoral pain syndrome subjects. Computer Methods in Biomechanics and Biomedical Engineering. 18(4):391-400.

Johnson JE, McIff TE, Lee P, Toby EB, Fischer KJ. 2014. Validation of radiocarpal joint contact models based on images from a clinical mri scanner. Computer Methods in Biomechanics and Biomedical Engineering. 17(4):378-387.

Khalil W, Dombre E. 2004. Modeling, identification and control of robots. ButterworthHeinemann.

Krings M, Nyakatura JA, Boumans ML, Fischer MS, Wagner H. 2017. Barn owls maximize head rotations by a combination of yawing and rolling in functionally diverse regions of the neck. Journal of anatomy. 231(1):12-22.

Nyakatura JA, Allen VR, Lauströer J, Andikfar A, Danczak M, Ullrich HJ, Hufenbach W, Martens T, Fischer MS. 2015. A three-dimensional skeletal reconstruction of the stem amniote orobates pabsti (diadectidae): analyses of body mass, centre of mass position, and joint mobility. PLoS One. 10(9):e0137284.

Ottoboni A, Parenti-Castelli V, Sancisi N, Belvedere C, Leardini A. 2010. Articular surface approximation in equivalent spatial parallel mechanism models of the human knee joint: an 
experiment-based assessment. Proceedings of the Institution of Mechanical Engineers, Part H: Journal of Engineering in Medicine. 224(9):1121-1132.

Parenti-Castelli V, Leardini A, Di Gregorio R, O'connor JJ. 2004. On the modeling of passive motion of the human knee joint by means of equivalent planar and spatial parallel mechanisms. Autonomous Robots. 16(2):219-232.

Robert E Kambic AAB, Pierce SE. 2017. Experimental determination of three-dimensional cervival mobility in the avian neck. Frontier in Zoology (2017).

Siegler S, Konow T, Belvedere C, Ensini A, Kulkarni R, Leardini A. 2018. Analysis of surfaceto-surface distance mapping during three-dimensional motion at the ankle and subtalar joints.

Terray L, Plateau O, Abourachid A, Böhmer C, Delapre A, De La Bernardie X, Cornette R. 2019. Modularity and diversity of the bird's neck: a 3D-geometric morphometric approach of cervical vertebrae. In: International Congress of Vertebrate Morphology (ICVM); vol. 280; Jun; Prague, Czech Republic. p. S228. 